\title{
STUDIES WITH RADIOACTIVE ENDOTOXIN. II. CORRELATION OF PHYSIO- LOGIC EFFECTS WITH DISTRIBUTION OF RADIOACTIVITY IN RABBITS INJECTED WITH LETHAL DOSES OF E. COLI ENDOTOXIN LABELLED WITH RADIOACTIVE SODIUM CHROMATE ${ }^{1}$
}

\author{
By A. I. BRAUDE, F. J. CAREY, AND MARGARET ZALESKY \\ (From the Department of Internal Medicine, The University of Texas, Southwestern Medical \\ School, Dallas, Texas)
}

(Submitted for publication December 27, 1954; accepted February 23, 1955)

The development of a simple technique for labelling $E$. coli endotoxin with radioactive chromium has presented the opportunity for tracer experiments (1). Such experiments have been conducted in different species of animals, using various doses of endotoxin tagged with either hexavalent chromium as $\mathrm{Na}_{2} \mathrm{Cr}^{51} \mathrm{O}_{4}$, or trivalent chromium, as $\mathrm{Cr}^{51} \mathrm{Cl}_{8}$. One of the objectives was to observe the physiologic changes which unfold as the endotoxin is removed from the blood and deposited in various organs. This paper presents the first of a series of studies describing these changes; it deals with the injection into rabbits of lethal doses of endotoxin tagged with hexavalent radiochromium.

\section{METHODS}

Endotoxin, prepared from bacteria grown in synthetic medium, was labelled by direct incubation with $\mathrm{Na}_{2} \mathrm{Cr}^{51} \mathrm{O}$, by the method described in the preceding paper (1). When injected into rabbits, there were 223.4 counts per second per mg. and the $\mathrm{LD}_{\mathrm{so}}$ for mice before and after tagging was $0.2 \mathrm{mg}$.

Three groups of adult rabbits weighing 2.5 to $3.0 \mathrm{~kg}$. were injected intravenously with $\mathrm{Cr}^{\mathrm{s1}}$ as follows:

Group I-Endotoxin labelled with $\mathrm{Na}_{2} \mathrm{Cr}^{\mathrm{In}} \mathrm{O}_{4}$.

Group II- $\mathrm{Na}_{2} \mathrm{Cr}^{\mathrm{CO}} \mathrm{O}_{4}$ in one vein followed by the injection of unlabelled endotoxin in another vein.

Group III- $\mathrm{Na}_{2} \mathrm{Cr}^{m} \mathrm{O}_{4}$ only (no endotoxin).

The dose of endotoxin and chromium is given for each group in Tables IA, IB, IC and Figures 1A, 1B, 1C. The dose of radioactive endotoxin was selected after determining from preliminary experiments: 1) that the pattern of distribution of radioactivity was the same over a wide range of lethal doses, and 2) that the amount of radioactive endotoxin injected in these experiments was enough to provide accurate counts in the tissues without killing the animals in too short a time to complete the experiment.

1 This investigation was supported in part by the Research and Development Division, Office of The Surgeon General, Department of the Army, under Contract No. DA-49-007-MD-519.
Before the injection of endotoxin, leukocyte counts were made on blood from the ear vein, and rectal temperatures and blood pressures were measured repeatedly until the rabbits were stabilized. The blood pressures were measured by placing a human blood pressure cuff around the abdomen, inflating it in the usual manner, and palpating the femoral arterial pulse until it was obliterated. This method for measuring blood pressure in rabbits has been checked by simultaneous determinations with the ear-chamber apparatus of Grant and Rothschild (2), and the results were identical when the chamber and cuff were connected to the same manometer. Although the abdominal-cuff method may not be as precise as direct intra-arterial measurement, it provides a simple and satisfactory non-surgical technique for demonstrating changes in pressure, and does not require anesthesia which might complicate the studies on endotoxin.

When the animals were about to be sacrificed, determinations of blood pressure, temperature, and leukocyte count were repeated, and 30 to $50 \mathrm{ml}$. of blood was drawn from the heart. The blood was citrated with one-fourth its volume of ACD (1.32 gm. sodium citrate, $0.48 \mathrm{gm}$. citric acid, and $1.47 \mathrm{gm}$. dextrose per $100 \mathrm{ml}$.) solution and separated into its three components; plasma, red cells, and buffy coat. The plasma was easily removed after centrifugation at 5,000 r.p.m. in the refrigerated ultracentrifuge for 20 minutes. Buffy coat was next removed in the form of a continuous membrane with a Pasteur pipette. The buffy coat and accompanying red cells were suspended in saline in a small test tube ( $75 \times$ $9 \mathrm{~mm}$ ) and centrifuged at approximately 2,000 r.p.m. for 10 minutes. After several washings with saline, the buffy coat had been freed almost entirely of red cells.

Animals were sacrificed by exsanguination, during which process saline was injected intravenously so that the animals remained alive long enough to permit removal of maximum amounts of blood.

The tissues of all sacrificed animals were weighed and then soaked in tap water at $4^{\circ} \mathrm{C}$. until the washings contained no appreciable hemoglobin in a 24-hour period. This procedure was necessary to insure that the presence of radioactivity in an organ was not that of the blood contained within it. Admittedly, endotoxin could be lost from the tissues but it is unlikely that a large proportion would disappear in view of the finding that the total radioactivity in the combined washings did not exceed by more than 5 per cent of the total injected that calculated to be present in the blood which had been washed 
TABLE IA

GROUP I

\section{Concentration of Radioactivity in Various Tissues $=\frac{\mathbf{x} \text { TOTAL INJECTED ACTNVITY }}{\mathrm{q} \text { TOTAL BODY WEIGHT }}$}

\begin{tabular}{|c|c|c|c|c|c|c|c|c|c|c|c|c|}
\hline 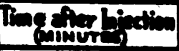 & & -15 & & 15 & 8 & 5 & & 105 & & 140 & 225 & 240 \\
\hline Rabbit No. & 27 & 28 & 85 & 36 & 32 & 30 & 25 & 31 & 26 & 29 & 38 & 34 \\
\hline $\begin{array}{l}\text { BLOOD: } \\
\text { Red Colls }\end{array}$ & 0 & 0 & & $\begin{array}{l}.11 \\
.32 \\
.30\end{array}$ & 0 & 0 & $\begin{array}{c}.1 \\
.34 \\
.3\end{array}$ & 0 & & $\begin{array}{l}\frac{1.1}{.63} \\
1.7\end{array}$ & 0 & $\begin{array}{l}\frac{1.1}{7.4} \\
1.4\end{array}$ \\
\hline Plasma & \begin{tabular}{|l|}
$\frac{23.6}{1.86}$ \\
12.69 \\
\end{tabular} & \begin{tabular}{|l|}
$\frac{23.8}{1.77}$ \\
13.46 \\
\end{tabular} & & \begin{tabular}{|l|}
$\frac{6.13}{685}$ \\
7.21 \\
\end{tabular} & \begin{tabular}{|l|}
$\frac{9.2}{.7}$ \\
13.1 \\
\end{tabular} & $\begin{array}{c}\frac{7.86}{1.12} \\
7\end{array}$ & $\begin{array}{l}.11 \\
.58 \\
6.15 \\
\end{array}$ & \begin{tabular}{|c|}
$\frac{8.6}{1.01}$ \\
8 \\
\end{tabular} & & $\begin{array}{c}2.3 \\
1.36 \\
9\end{array}$ & $\begin{array}{l}3.3 \\
.7 \\
4.7\end{array}$ & \\
\hline Buffy $\cos$ & \begin{tabular}{|l|}
$\frac{2.87}{.00012}$ \\
7000 \\
\end{tabular} & \begin{tabular}{|l|}
$\frac{1.32}{.0012}$ \\
1200 \\
\end{tabular} & & \begin{tabular}{|l|}
2.69 \\
00021 \\
12,000
\end{tabular} & \begin{tabular}{|l|}
2.76 \\
25,0000 \\
25,000
\end{tabular} & & \begin{tabular}{|l|}
$\frac{2.98}{.00,55}$ \\
18,000 \\
\end{tabular} & \begin{tabular}{|l|}
.6 \\
.005 \\
200
\end{tabular} & & $\begin{array}{l}.54 \\
.00000 \\
6.000\end{array}$ & $\begin{array}{c}\frac{277}{.005} \\
10,000\end{array}$ & $\begin{array}{l}\frac{2.81}{.004} \\
6,000\end{array}$ \\
\hline LIVER & \begin{tabular}{|l|}
$\frac{9.22}{3.32}$ \\
2.77 \\
\end{tabular} & \begin{tabular}{|l|}
$\frac{9.89}{2.55}$ \\
3.87 \\
\end{tabular} & $\begin{array}{l}\frac{17.5}{3.5} \\
4.99 \\
\end{array}$ & \begin{tabular}{|l|}
16.26 \\
3.29 \\
4.94 \\
\end{tabular} & \begin{tabular}{|l|}
$\frac{19.8}{2.57}$ \\
5.11 \\
\end{tabular} & $\begin{array}{l}\frac{14.08}{2.5} \\
5.6 \\
\end{array}$ & \begin{tabular}{|l|}
11.79 \\
2.8 \\
4.18 \\
\end{tabular} & \begin{tabular}{|l|}
$\frac{15.6}{3.8}$ \\
4.1 \\
\end{tabular} & \begin{tabular}{c|}
17 \\
3.57 \\
5.05 \\
\end{tabular} & $\begin{array}{l}17.03 \\
3.72 \\
4.56 \\
\end{array}$ & & $\begin{array}{l}\frac{17.02}{3.28} \\
5.19 \\
\end{array}$ \\
\hline$\underline{L}$ & \begin{tabular}{|l|}
2.85 \\
.55 \\
5.18 \\
\end{tabular} & \begin{tabular}{|l|}
4.87 \\
9.57 \\
9.51 \\
\end{tabular} & $\begin{array}{l}\frac{1.9}{4.2} \\
4.5\end{array}$ & $\begin{array}{l}.14 \\
4.39 \\
\end{array}$ & \begin{tabular}{|l|}
5.3 \\
4.7 \\
11.3 \\
\end{tabular} & $\begin{array}{l}\frac{2.6}{6} \\
4.3\end{array}$ & $\begin{array}{l}\frac{6.99}{.77} \\
2.07 \\
\end{array}$ & $\begin{array}{l}2.95 \\
6.4 \\
4.6 \\
\end{array}$ & \begin{tabular}{|l|}
2.2 \\
.8 \\
2.75 \\
\end{tabular} & \begin{tabular}{|l|}
2.2 \\
.59 \\
3.76
\end{tabular} & $\begin{array}{l}\frac{1.42}{4.48} \\
2.95\end{array}$ & $\begin{array}{l}\frac{.23}{.34} \\
0.6 \\
\end{array}$ \\
\hline HEART & \begin{tabular}{|l|}
.26 \\
.27 \\
.96
\end{tabular} & $\begin{array}{l}\frac{.7}{.24} \\
.70\end{array}$ & $\begin{array}{l}\frac{.42}{.25} \\
1.68\end{array}$ & \begin{tabular}{|l|}
.37 \\
.28 \\
1.32 \\
\end{tabular} & $\begin{array}{l}.05 \\
.22 \\
.22\end{array}$ & $\begin{array}{l}\frac{.22}{24} \\
.91\end{array}$ & \begin{tabular}{|l|}
.37 \\
.28 \\
1.32 \\
\end{tabular} & \begin{tabular}{l|}
.36 \\
.20 \\
1.2
\end{tabular} & $\begin{array}{c}.36 \\
.40 \\
.9\end{array}$ & $\begin{array}{l}\frac{.18}{.37} \\
.49\end{array}$ & $\begin{array}{l}\frac{.28}{.35} \\
0.8\end{array}$ & $\begin{array}{l}\frac{94}{687} \\
1.1\end{array}$ \\
\hline KIDNEY & \begin{tabular}{|l|}
.85 \\
.66 \\
1.28 \\
\end{tabular} & & $\begin{array}{l}\frac{8}{.65} \\
1.23 \\
\end{array}$ & \begin{tabular}{|l|}
$\frac{1.16}{.73}$ \\
1.58 \\
\end{tabular} & $\begin{array}{l}.37 \\
.71 \\
1.9 \\
\end{array}$ & $\begin{array}{l}.33 \\
.52 \\
.6 \\
\end{array}$ & \begin{tabular}{|l|}
3.18 \\
.63 \\
5.04 \\
\end{tabular} & $\begin{array}{l}\frac{.77}{.74} \\
1.04 \\
\end{array}$ & $\begin{array}{l}2.59 \\
.64 \\
4.05 \\
\end{array}$ & $\begin{array}{l}.92 \\
.61 \\
1.5 \\
\end{array}$ & & \\
\hline EN & \begin{tabular}{|l|}
.22 \\
.07 \\
3.14 \\
\end{tabular} & \begin{tabular}{|l|}
.15 \\
.06 \\
2.5 \\
\end{tabular} & & \begin{tabular}{|l|}
.31 \\
.04 \\
7.75 \\
\end{tabular} & $\begin{array}{l}\frac{.31}{.04} \\
7.75 \\
\end{array}$ & & $\begin{array}{l}\frac{.21}{.06} \\
3.5 \\
\end{array}$ & $\begin{array}{l}. .2 \\
.06 \\
3.3\end{array}$ & $\begin{array}{c}\frac{.72}{.05} \\
8 \\
\end{array}$ & $\begin{array}{l}\frac{.2}{.06} \\
3.3\end{array}$ & $\begin{array}{l}\frac{.34}{.05} \\
6.8 \\
\end{array}$ & \\
\hline STINE & \begin{tabular}{|l|}
1.87 \\
3.64 \\
.51 \\
\end{tabular} & \begin{tabular}{|l|}
$\frac{1.15}{4.7}$ \\
.24 \\
\end{tabular} & $\begin{array}{l}\frac{1.47}{3.31} \\
.27 \\
\end{array}$ & \begin{tabular}{|l|}
4.2 \\
.86 \\
\end{tabular} & & 0 & & $\begin{array}{l}.3 \\
2.8 \\
0.5 \\
\end{array}$ & \begin{tabular}{|c|}
$\frac{3.2}{4.64}$ \\
.68 \\
\end{tabular} & & $\begin{array}{c}\frac{1.47}{6.68} \\
0.2\end{array}$ & $\begin{array}{l}\frac{1.48}{6.45} \\
.22 \\
\end{array}$ \\
\hline Muscle & \begin{tabular}{|c|}
$\frac{2.35}{5.46}$ \\
.43 \\
\end{tabular} & \begin{tabular}{|l|}
$\frac{1.15}{4.53}$ \\
.25 \\
\end{tabular} & & \begin{tabular}{|c|}
.73 \\
4.98 \\
.14 \\
\end{tabular} & $\begin{array}{l}\frac{.73}{4} \\
.18 \\
\end{array}$ & & & 0 & & \begin{tabular}{|c|}
$\frac{1.09}{5.20}$ \\
.2 \\
\end{tabular} & 0 & $\begin{array}{l}\frac{1.32}{4.62} \\
.29 \\
\end{array}$ \\
\hline BONE & & $\begin{array}{l}.56 \\
1.38 \\
\end{array}$ & & \begin{tabular}{l|}
$\frac{.86}{.56}$ \\
1.53 \\
\end{tabular} & & $\begin{array}{l}\frac{.79}{.59} \\
1.46\end{array}$ & \begin{tabular}{|l|}
1.27 \\
.67 \\
1.89 \\
\end{tabular} & $\begin{array}{l}.73 \\
.55 \\
1.32 \\
\end{array}$ & $\begin{array}{l}1.19 \\
.46 \\
2.48 \\
\end{array}$ & \begin{tabular}{|l|}
.91 \\
.43 \\
2.1 \\
\end{tabular} & \begin{tabular}{|l|}
.96 \\
.75 \\
1.3 \\
\end{tabular} & $\begin{array}{l}\frac{.96}{.45} \\
2.13\end{array}$ \\
\hline
\end{tabular}

Each rabbit received $6 \mathrm{mg}$. endotoxin containing 223.4 counts per second par $\mathrm{mg}$.

out. After washing, tissues were dried in an oven at $100^{\circ} \mathrm{C}$. The muscle and intestine were incinerated to ashes at $500^{\circ} \mathrm{C}$. and the liver, lung, heart, kidney, and spleen were digested with concentrated nitric acid. The femurs were dried and pulverized. Plasma, red cells, and buffy coats were weighed but not digested. Total weights per animal of plasma, red cells, and buffy coats were calculated from the weights of the samples examined on the basis of blood volume determinations made in normal rabbits of equivalent size as described below.

The radioactivity of the tissues and injected chromium was determined as previously described in the scintillation counter after counting for 10 minutes (1). Loss of activity resulting from lapse of time during treatment of tissues was accounted for by plotting the time-decay curve.

Observations were also made on blood volume following the injection of endotoxin. Because the method for measuring blood volume was that of tagging red cells with $\mathrm{Cr}^{\circ}$, it was necessary to inject non-labelled endotoxin. Blood volume studies were conducted in three rabbits receiving endotoxin. Their red cells were obtained for labelling by drawing $20 \mathrm{ml}$. of heart blood from each into $5 \mathrm{ml}$. of ACD solution. The red cells were separated by centrifugation at 2,000 r.p.m. for $15 \mathrm{~min}$ utes and then the plasma supernate was removed. To 
each sample of red cells were added 200 microcuries in $.103 \mathrm{mg}$. of $\mathrm{Na}_{2} \mathrm{Cr}^{51} \mathrm{O}_{4}$. The mixture of red cells and chromium was incubated at $37^{\circ} \mathrm{C}$. for one hour with frequent gentle agitation. Then the red cells were washed with saline three times, resuspended in their own plasma, and injected back into the rabbit from which they had been removed. The process required a total of 4 to 5 hours. The tagged cells were allowed to circulate for 72 hours before endotoxin was injected because the animals were unduly susceptible to endotoxin if it was injected within a few hours after the tagged cells had been returned to the rabbits.

To determine blood volumes, $4.0 \mathrm{ml}$. of blood were removed from the animal's heart and the activity determined in a well-type gamma ray counter manufac- tured by the Texas Company. Blood volume equalled total counts injected

counts per sec. per ml. of circulating blood. After each rabbit's normal blood volume was determined, the rabbit was given intravenously $1.0 \mathrm{mg}$. of non-labelled endotoxin. Blood samples of $4.0 \mathrm{ml}$. were removed from the heart at various intervals up to the time of death and blood pressures were determined periodically. Hematocrits were measured on the $4.0 \mathrm{ml}$. of cardiac heparinized blood, and leukocytes were counted in the blood from the ear vein. Red cell mass was calculated by multiplying hematocrit times blood volume and corrections were made for those cells removed at the time of each blood volume determination.

To test the distribution in vitro of free chromium and

TABLE IB

GROUP II

\section{Concentration of Radioactivity in Various Tissues $=\frac{7 \text { TOTAL INJECTED ACTNITY }}{7 \text { TOTAL BODY WEIGHT }}$}

\begin{tabular}{|c|c|c|c|c|c|c|c|c|c|c|c|c|c|}
\hline \multirow{2}{*}{$\begin{array}{l}\text { Iing after lajection } \\
\text { (abbutt No. } \\
\text { Rabbit No. }\end{array}$} & \multicolumn{3}{|c|}{$0-10$} & \multicolumn{2}{|c|}{40} & \multirow{2}{*}{\begin{tabular}{c|}
60 \\
7
\end{tabular}} & \multirow{2}{*}{$\frac{75}{8}$} & \multicolumn{2}{|c|}{90} & \multirow{2}{*}{\begin{tabular}{|c|}
120 \\
10 \\
\end{tabular}} & \multirow{2}{*}{\begin{tabular}{|c|}
150 \\
4 \\
\end{tabular}} & \multicolumn{2}{|c|}{330} \\
\hline & 1 & 2 & 3 & 6 & 13 & & & 9 & 5 & & & II & 12 \\
\hline $\begin{array}{l}\text { BLOOD: } \\
\text { Red Cells }\end{array}$ & $\begin{array}{l}\frac{5.33}{0.3} \\
16.2\end{array}$ & \begin{tabular}{l|}
$\frac{3.88}{0.43}$ \\
9.02
\end{tabular} & & $\begin{array}{l}\frac{8.43}{0.46} \\
18.3\end{array}$ & $\begin{array}{l}\frac{550}{0.34} \\
15.94\end{array}$ & $\begin{array}{l}\frac{3.24}{0.41} \\
7.9\end{array}$ & & & $\begin{array}{l}\frac{6.3}{0.49} \\
12.8\end{array}$ & $\begin{array}{l}\frac{3.55}{0.26} \\
15.2\end{array}$ & \begin{tabular}{|l|}
$\frac{3.1}{0.41}$ \\
256
\end{tabular} & \begin{tabular}{|l|}
$\frac{4.6}{0.44}$ \\
$10: 45$
\end{tabular} & $\begin{array}{l}\frac{2.5}{0.14} \\
178\end{array}$ \\
\hline Plasma & $\begin{array}{l}\frac{2.83}{1.12} \\
2.5 \\
\end{array}$ & $\begin{array}{l}3.19 \\
1.73 \\
1.84 \\
\end{array}$ & & \begin{tabular}{|l|}
1.78 \\
0.07 \\
\end{tabular} & \begin{tabular}{|l|}
1.78 \\
0.79 \\
2.25 \\
\end{tabular} & $\begin{array}{l}1.94 \\
0.83 \\
2.333 \\
\end{array}$ & & & $\begin{array}{l}1.98 \\
0.28 \\
2.25 \\
\end{array}$ & $\begin{array}{l}0.91 \\
0.60 \\
1.52\end{array}$ & \begin{tabular}{l|}
1.7 \\
0.64 \\
2.65 \\
\end{tabular} & $\begin{array}{l}\frac{a 71}{a 58} \\
1.26 \\
\end{array}$ & $\begin{array}{l}\frac{0.55}{0.20} \\
1.25 \\
\end{array}$ \\
\hline & $\begin{array}{l}\frac{8.86}{3.1} \\
2.89 \\
\end{array}$ & \begin{tabular}{l|}
7.3 \\
2.79 \\
2.6 \\
\end{tabular} & \begin{tabular}{|l|}
$\frac{6.3}{3.09}$ \\
2.03 \\
\end{tabular} & $\begin{array}{l}\frac{4.93}{2.55} \\
1.95 \\
\end{array}$ & $\begin{array}{l}4: 31 \\
3.38 \\
1.13 \\
\end{array}$ & $\begin{array}{l}\frac{6.21}{2.61} \\
2.37 \\
\end{array}$ & $\begin{array}{l}\frac{5.31}{2.15} \\
2.47 \\
\end{array}$ & $\begin{array}{l}\frac{5.31}{3.23} \\
1.64 \\
\end{array}$ & $\begin{array}{l}\frac{4.6}{3.45} \\
1.3 \\
\end{array}$ & $\begin{array}{l}\frac{6.37}{3.22} \\
1.95 \\
\end{array}$ & \begin{tabular}{|l|}
$\frac{5.3}{204}$ \\
2.59 \\
\end{tabular} & $\begin{array}{l}\frac{5.4}{2.56} \\
2.28\end{array}$ & $\begin{array}{l}3.3 \\
2.44 \\
1.58 \\
\end{array}$ \\
\hline & & $\begin{array}{l}1.03 \\
0.78 \\
1.32 \\
\end{array}$ & $\begin{array}{l}\frac{0.43}{0.65} \\
0.66\end{array}$ & $\begin{array}{l}\frac{0.67}{0.67} \\
1.0\end{array}$ & $\begin{array}{l}\frac{0.87}{0.53} \\
1.56\end{array}$ & $\begin{array}{l}\frac{0.87}{0253} \\
\text { L64 }\end{array}$ & $\begin{array}{l}\frac{0.85}{1.06} \\
0.80\end{array}$ & $\begin{array}{l}\frac{0.89}{057} \\
1.32\end{array}$ & $\begin{array}{l}\frac{1.10}{0.61} \\
1.80\end{array}$ & $\begin{array}{l}\frac{0.57}{0.59} \\
1.13\end{array}$ & \begin{tabular}{|l|}
$\frac{0.8}{\alpha .44}$ \\
1.62
\end{tabular} & $\begin{array}{l}\frac{0.2}{0.33} \\
2.18\end{array}$ & $\begin{array}{l}\frac{0.62}{0.62} \\
1.0\end{array}$ \\
\hline & $\begin{array}{l}\frac{0.49}{0.23} \\
2.13\end{array}$ & \begin{tabular}{l|}
0.31 \\
0.32 \\
0.97 \\
\end{tabular} & $\begin{array}{l}0.97 \\
0.30 \\
2.23\end{array}$ & $\begin{array}{l}\frac{0.26}{0.3 I} \\
0.83\end{array}$ & $\begin{array}{l}\frac{0.25}{0.21} \\
.86 \\
\end{array}$ & $\begin{array}{l}\frac{0.19}{d .30} \\
0.63\end{array}$ & $\begin{array}{l}\frac{0.9}{0.41} \\
0.53\end{array}$ & $\begin{array}{l}\frac{0.53}{0.40} \\
0.8\end{array}$ & $\begin{array}{l}\frac{0.45}{0.34} \\
1.3\end{array}$ & $\begin{array}{l}0.36 \\
0.59 \\
0.92\end{array}$ & \begin{tabular}{|l|}
0.4 \\
0.26 \\
1.5 \\
\end{tabular} & \begin{tabular}{l|}
0.26 \\
0.35 \\
0.74
\end{tabular} & $\begin{array}{l}\frac{0.13}{0.25} \\
0.62\end{array}$ \\
\hline & $\begin{array}{l}\frac{3.08}{0.62} \\
4.9 \\
\end{array}$ & \begin{tabular}{l|}
3.5 \\
.76 \\
4.6 \\
\end{tabular} & $\begin{array}{l}1.98 \\
0.59 \\
3.35 \\
\end{array}$ & $\begin{array}{l}\frac{6.49}{0.72} \\
9.01\end{array}$ & $\begin{array}{l}\frac{4.53}{0.59} \\
8.44\end{array}$ & $\begin{array}{l}\frac{9.31}{0.61} \\
15.5\end{array}$ & $\begin{array}{l}\frac{6.5}{0.54} \\
12.0\end{array}$ & $\begin{array}{l}\frac{6.89}{0.62} \\
11.1\end{array}$ & $\begin{array}{l}\frac{295}{0.73} \\
1363\end{array}$ & & \begin{tabular}{|l|}
$\frac{8.1}{0.11}$ \\
15.88 \\
\end{tabular} & \begin{tabular}{|l|}
2.49 \\
0.63 \\
15.06 \\
\end{tabular} & $\begin{array}{l}\frac{10.5}{0.56} \\
13.7\end{array}$ \\
\hline SPLEEN & & $\begin{array}{l}0.12 \\
0.05 \\
2.4 \\
\end{array}$ & \begin{tabular}{|l|}
0.07 \\
.04 \\
1.75 \\
\end{tabular} & $\begin{array}{l}\frac{0.10}{0.03} \\
3.33\end{array}$ & $\begin{array}{l}\frac{0.12}{0.08} \\
1.5\end{array}$ & $\begin{array}{l}.95 \\
.96 \\
0.7 \\
\end{array}$ & & $\begin{array}{l}\frac{0.10}{0.08} \\
1.25\end{array}$ & $\begin{array}{l}\frac{.07}{0.04} \\
1.75\end{array}$ & $\begin{array}{l}\frac{0.25}{.05} \\
5.0\end{array}$ & \begin{tabular}{|l|}
0.73 \\
0.06 \\
12.3 \\
\end{tabular} & $\begin{array}{l}\frac{1.23}{0.04} \\
32\end{array}$ & $\begin{array}{l}\frac{.07}{0.05} \\
1.42\end{array}$ \\
\hline ESTINE & $\begin{array}{l}\frac{2.57}{4.74} \\
0.54\end{array}$ & - & & $\begin{array}{l}\frac{2.21}{4.77} \\
0.46\end{array}$ & $\begin{array}{l}\frac{1.10}{3.02} \\
0.36\end{array}$ & $\begin{array}{l}0.52 \\
5.54 \\
0.96\end{array}$ & $\begin{array}{l}\frac{1.5}{4.78} \\
0.31\end{array}$ & & & $\begin{array}{l}\frac{2 A 1}{4 A B} \\
0.53\end{array}$ & & \begin{tabular}{|l|}
0.55 \\
0.19 \\
0.19 \\
\end{tabular} & $\begin{array}{l}\frac{1.44}{4.13} \\
0.27\end{array}$ \\
\hline MUSCLE & $\begin{array}{l}.21 \\
5.65 \\
0.39\end{array}$ & & $\begin{array}{l}2.57 \\
.20 \\
0.61 \\
\end{array}$ & $\begin{array}{l}\frac{1.03}{5.74} \\
0.17\end{array}$ & $\begin{array}{l}\frac{2.21}{6.29} \\
0.35\end{array}$ & $\begin{array}{l}\frac{2.55}{a .83} \\
3.26\end{array}$ & \begin{tabular}{l|}
1.10 \\
5.63 \\
0.19 \\
\end{tabular} & & & $\begin{array}{l}\frac{1.66}{5.03} \\
0.33\end{array}$ & & \begin{tabular}{l|}
1.59 \\
5.66 \\
0.24
\end{tabular} & $\begin{array}{l}\frac{1.10}{5.04} \\
0.22 \\
\end{array}$ \\
\hline BONE & $\begin{array}{l}\frac{0.71}{0.77} \\
0.92\end{array}$ & & $\begin{array}{l}0.74 \\
0.66 \\
1.12\end{array}$ & $\begin{array}{l}\frac{1.19}{0.71} \\
1.67\end{array}$ & $\begin{array}{l}\frac{1.01}{0.48} \\
2.08\end{array}$ & & $\begin{array}{l}0.87 \\
0.54 \\
1.61\end{array}$ & $\begin{array}{l}\frac{1.35}{0.5} \\
2.7\end{array}$ & & $\begin{array}{l}\frac{0.74}{0.62} \\
1.19\end{array}$ & \begin{tabular}{|l|}
$\frac{0.9}{0.45}$ \\
2.0
\end{tabular} & $\begin{array}{l}\frac{0.55}{0.49} \\
1.14\end{array}$ & $\begin{array}{l}\frac{0.68}{0.47} \\
1.45\end{array}$ \\
\hline
\end{tabular}

Each rabbit received $5 \mathrm{mg}$. endotoxin in one vein and $2 \mathrm{ml}$. of $\mathrm{Na}_{2} \mathrm{CrO}_{4}$ solution containing 2506 counts/second/ $\mathrm{ml}$. in another vein. 
TABLE IC GROUP III

\section{Concentration of Radioactivity in Various Tissues $=\frac{\% \text { TOTAL INUECTED ACTVITY }}{\text { \& TOTAL BODY WEIGHT }}$}

\begin{tabular}{|c|c|c|c|c|c|c|c|c|c|c|c|}
\hline 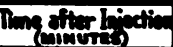 & \multicolumn{2}{|c|}{$0-5$} & \multicolumn{3}{|c|}{60} & \multicolumn{2}{|c|}{90} & \multicolumn{2}{|c|}{140} & 360 & 370 \\
\hline Rabbit Na & 14 & 15 & 16 & 20 & 21 & 17 & 22 & 18 & 19 & 23 & 24 \\
\hline $\begin{array}{l}\text { BLOOD: } \\
\text { Red Cells }\end{array}$ & $\frac{2.78}{.21}$ & & $\begin{array}{l}\frac{5.03}{.64} \\
5.98\end{array}$ & $\frac{7}{0.44}$ & $\begin{array}{l}\frac{4.72}{.6} \\
7.2\end{array}$ & $\begin{array}{l}\frac{5.76}{0.88} \\
6.54\end{array}$ & $\begin{array}{l}\frac{5.76}{0.77} \\
7.48\end{array}$ & $\frac{5.41}{.77}$ & $\frac{5.41}{5.44}$ & $\frac{1.41}{1.14}$ & 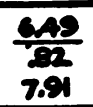 \\
\hline Plasma & & & $\begin{array}{l}3.95 \\
1.41 \\
2.80\end{array}$ & $\begin{array}{l}\frac{2.06}{1.66} \\
1.24\end{array}$ & $\begin{array}{l}\frac{306}{1.6} \\
1.0\end{array}$ & & $\begin{array}{l}\frac{4.32}{1.81} \\
2.38\end{array}$ & $\begin{array}{l}\frac{287}{1.77} \\
1.62\end{array}$ & $\begin{array}{l}\frac{1.62}{1.80} \\
0.80\end{array}$ & $\begin{array}{l}\frac{1.78}{1605} \\
.95\end{array}$ & $\frac{1.73}{1.53}$ \\
\hline LIVER & $\begin{array}{l}\frac{4.63}{3.26} \\
1.42\end{array}$ & $\begin{array}{l}\frac{3.24}{2.35} \\
1.37\end{array}$ & $\begin{array}{l}\frac{6.63}{2.22} \\
298\end{array}$ & $\begin{array}{l}\frac{5.31}{3.41} \\
1.55\end{array}$ & & $\begin{array}{l}\frac{371}{344} \\
.96\end{array}$ & $\begin{array}{l}\frac{4.03}{2.77} \\
1.45\end{array}$ & $\begin{array}{l}\frac{4.64}{2.45} \\
1.89\end{array}$ & $\begin{array}{l}\frac{8.96}{2.16} \\
4.14\end{array}$ & $\begin{array}{l}\frac{5.71}{3.02} \\
1.75\end{array}$ & $\begin{array}{l}\frac{995}{259} \\
399\end{array}$ \\
\hline LUNG & $\begin{array}{l}\frac{1.42}{.68} \\
2.08\end{array}$ & $\begin{array}{l}.98 \\
.48 \\
2.04\end{array}$ & $\begin{array}{l}1.27 \\
0.57 \\
2.22\end{array}$ & $\begin{array}{l}0.88 \\
0.77 \\
1.14\end{array}$ & & $\begin{array}{l}\frac{1.42}{.60} \\
2.36\end{array}$ & $\begin{array}{l}\frac{1.06}{.61} \\
1.73\end{array}$ & $\begin{array}{l}\frac{0.95}{0.52} \\
1.82\end{array}$ & $\frac{\frac{92}{.41}}{2.24}$ & & $\frac{.95}{.62}$ \\
\hline HEARt & $\begin{array}{l}\frac{.31}{.27} \\
1.14\end{array}$ & $\begin{array}{l}. .22 \\
.23 \\
.95\end{array}$ & $\begin{array}{l}\frac{.34}{.23} \\
1.47 \\
\end{array}$ & $\begin{array}{l}\frac{0.32}{0.36} \\
.88\end{array}$ & & $\begin{array}{l}\frac{.31}{.20} \\
1.06\end{array}$ & $\begin{array}{l}\frac{.50}{.20} \\
1.72\end{array}$ & $\begin{array}{l}\frac{96}{31} \\
1.16\end{array}$ & $\begin{array}{l}\frac{0.42}{.50} \\
1.40\end{array}$ & $\begin{array}{l}\frac{28}{.35} \\
.80\end{array}$ & \\
\hline KIDNEY & $\begin{array}{l}\frac{1.19}{.76} \\
1.58\end{array}$ & & $\begin{array}{l}\frac{8.32}{0.53} \\
15.1\end{array}$ & $\begin{array}{l}\frac{12.8}{.89} \\
14.38\end{array}$ & & $\begin{array}{l}\frac{9.5}{.50} \\
10.5\end{array}$ & $\begin{array}{l}\frac{6.36}{0.72} \\
8.8\end{array}$ & $\begin{array}{l}9.69 \\
0.60 \\
16.5\end{array}$ & $\begin{array}{c}\frac{.12}{.04} \\
3\end{array}$ & $\begin{array}{l}\frac{4.73}{35} \\
5.56\end{array}$ & $\frac{1.71}{5.0}$ \\
\hline SPLEEN & $\begin{array}{l}\frac{.25}{.17} \\
1.47\end{array}$ & $\begin{array}{l}\frac{.07}{.03} \\
2.33\end{array}$ & $\begin{array}{c}\frac{0.10}{0.02} \\
5\end{array}$ & $\begin{array}{l}.09 \\
.07 \\
1.28\end{array}$ & & $\begin{array}{l}\frac{.22}{.08} \\
2.75\end{array}$ & $\begin{array}{l}\frac{.09}{.08} \\
1.12\end{array}$ & $\begin{array}{l}\frac{0.09}{.10} \\
.90\end{array}$ & & $\frac{.12}{.05}$ & $\begin{array}{l}\frac{.48}{.04} \\
12\end{array}$ \\
\hline INTESTINE & & & & $\begin{array}{l}\frac{2.57}{6.42} \\
0.40\end{array}$ & & $\begin{array}{l}\frac{3.51}{5.56} \\
.65\end{array}$ & $\begin{array}{l}\frac{1.59}{5.53} \\
0.29\end{array}$ & & & & \\
\hline Muscle & & & $\begin{array}{l}\frac{1.42}{4.35} \\
0.32\end{array}$ & $\begin{array}{l}\frac{1.38}{6.18} \\
.22\end{array}$ & & & $\begin{array}{l}\frac{1.69}{6.45} \\
0.26\end{array}$ & & & & \\
\hline BONE & $\begin{array}{l}\frac{1.17}{.8} \\
1.46\end{array}$ & $\frac{.70}{.48}$ & $\begin{array}{l}. .79 \\
.53 \\
1.49\end{array}$ & $\begin{array}{l}\frac{.92}{.53} \\
1.73\end{array}$ & & $\begin{array}{l}\frac{1.55}{82} \\
180\end{array}$ & $\begin{array}{l}\frac{1.47}{0.83} \\
1.77\end{array}$ & $\begin{array}{l}\frac{1.03}{.60} \\
1.71\end{array}$ & & $\frac{1.09}{.64}$ & \\
\hline
\end{tabular}

Each rabbit received intravenously $2 \mathrm{ml}$. of $\mathrm{Na}_{2} \mathrm{CrO}_{4}$ containing 5012 counts/second/rotal

chromium bound to endotoxin each was mixed with citrated blood pooled from three rabbits as follows and incubated for one hour at $37^{\circ} \mathrm{C}$. :

$1.5 \mathrm{ml}$. $\mathrm{Na}_{2} \mathrm{Cr}^{61} \mathrm{O}_{4}$ containing 253 counts per second (Texas well counter) plus $105 \mathrm{ml}$. pooled blood.

$1.5 \mathrm{ml}$. labelled endotoxin (9 mg.) containing 253 counts per second plus $105 \mathrm{ml}$. pooled blood.

At the end of the incubation period, the bloods were separated into plasma, red cells, and buffy coat. Then each was examined for radioactivity. Cross matching was performed before the bloods were pooled to be certain that the experiment was not complicated by incompatibilities which might agglutinate red cells, or coat them with antibody in such a way that their affinity for chromium would be altered. Incompatibilities be- tween blood from rabbits have been repeatedly demonstrated in this laboratory.

\section{RESULTS}

The results are summarized in Tables IA, IB, and IC, and the corresponding Figures, 1A, 1B, and $1 \mathrm{C}$. It is clear that the distribution of radioactivity after injection of free chromium is entirely different from that after injection of tagged endotoxin. The most remarkable difference concerns the blood. Free chromium passes mainly to the red cells and only small amounts are in the plasma. Chromium attached to endotoxin, on the other 
hand, appears in the plasma in large quantities and virtually none goes to red cells. A striking amount of radioactivity appears in the buffy coat after injection of labelled endotoxin.

Among animals given labelled endotoxin, most of the activity in the tissues is found in the liver and only a small per cent in the kidneys. Animals receiving free chromium, by contrast, show heavy activity in the kidneys and urine, but relatively little in the liver. Rabbits receiving labelled endotoxin also show a greater uptake in the lung and spleen than those receiving free chromium. The increased uptake in spleen and lung is shown in
Table I which expresses the degree of concentration in tissues as well as per cent uptake. The relatively small size of certain viscera could permit marked concentration even though only a small per cent of total injected activity appears in them. Examinations of brain revealed no radioactivity after injection of labelled endotoxin.

The affinity of free $\mathrm{Cr}^{51}$ for red cells is also seen when $\mathrm{Na}_{2} \mathrm{Cr}^{51} \mathrm{O}_{4}$ is added to whole blood in vitro. Similarly, the failure of labelled endotoxin to appear in red cells is observed when radioactive endotoxin is mixed with whole blood. This is illustrated in the following results expressed as



Fig. 1A. Distribution of Radioactivity among Blood and Tissues of Rabbits InJECTED with RAdIOACTIVE ENDotoxin

The leukocyte count and blood pressure at time of sacrifice is expressed as per cent of the control value obtained in the same animal before endotoxin was injected. Each point represents a determination on one sacrificed rabbit. 




*Tatal Leveocyte count. of or Iginal.

Figure 1B

Symbols same as in 1A.



Frgure 1C

Symbols same as in $1 \mathrm{~A}$. 



FIGURe 2

Each rabbit received $1.0 \mathrm{mg}$. of non-labelled $E$. coli endotoxin intravenously at zero time.

counts per second (in the Texas well counter) of plasma, red cells, and buffy coat.

\begin{tabular}{|c|c|c|}
\hline & $\begin{array}{l}\text { Tagged endotoxin } \\
\text { plus whole blood }\end{array}$ & $\begin{array}{l}\text { Free chromium } \\
\text { plus whole blood }\end{array}$ \\
\hline $\begin{array}{l}\text { lasma } \\
\text { ed cells } \\
\text { uffy coat }\end{array}$ & $\begin{array}{c}224.8 \text { (87.3 per cent) } \\
1.28 \text { ( } 0.5 \text { per cent }) \\
0.5\end{array}$ & $\begin{array}{l}17.9 \\
186 \\
.06\end{array}\left(\begin{array}{l}7.07 \text { per cent }) \\
(73.5 \text { per cent })\end{array}\right.$ \\
\hline
\end{tabular}

Results of the studies on blood volume are given for the three rabbits in Figure 2.

If the tag on endotoxin with $\mathrm{Cr}^{51}$ can be regarded as stable in vivo, the results portrayed in Figures 1 and 2 may be summarized as follows: Endotoxin is carried in the plasma, but not red cells, immediately after intravenous injection. Within an hour, the plasma concentration of endotoxin falls to about $3 / 5$, and in two hours to $1 / 2$ of the original. The period of rapid removal from the plasma is accompanied by fever, leukopenia and sometimes by hypotension and diarrhea. As it leaves the plasma, the largest percentages of endotoxin go to the liver, and to the buffy coat. The buffy coat takes up a great amount of endotoxin despite its relatively minute total mass. The leukocyte count drops precipitously during the period when the endotoxin accumulates in the buffy coat. Both lymphocytes and neutrophils fall. During the first hour, as endotoxin leaves the plasma, there is an initial fall in blood pressure to nearly shock levels but this is usually compensated for, at least temporarily. Then it may fall again just before death. In other animals the blood pressure is maintained and the animal dies suddenly with cardiac arrest. The cardiac action of some dying animals is very weak and irregular even for an hour before death. This is best determined by palpation of the heart through the chest wall. The blood volume undergoes a variable response. In one animal, it remained remarkably constant; in a second, it showed a fall during a period of severe diarrhea and remained low; and in a third, there was an initial fall followed by a return toward normal in two hours.

\section{DISCUSSION}

There is a remarkable difference in distribution of radioactivity between that obtained after the injection of sodium chromate, and after endotoxin tagged with sodium chromate. This difference discloses the important fact that the $\mathrm{Cr}^{51}$ does not break away from endotoxin in vivo to follow the distribution of ionic hexavalent $\mathrm{Cr}^{51}$. Because of the high affinity of chromate ion for red cells, their radioactivity can be used as a simple but sensitive indicator of whether the experiment with labelled endotoxin is complicated by the injection, or the splitting-off in vivo, of free $\mathrm{Cr}^{51}$. This property of chromate ion is another reason why $\mathrm{Cr}^{51}$, in the form of sodium chromate, is well suited for the preparation of radioactive endotoxin. It must be emphasized, in this connection, that endotoxin does not reduce affinity of red cells for chromate ion. The radio- 
activity of red cells after the simultaneous injection of $\mathrm{Na}_{2} \mathrm{Cr}^{51} \mathrm{O}_{4}$ and non-labelled endotoxin (Figure 1B) was no less than that observed after $\mathrm{Na}_{2} \mathrm{Cr}^{51} \mathrm{O}_{4}$ was injected by itself.

Other experiments from this laboratory also support the validity of the chromium label for tracing the distribution of endotoxin in vivo. Essentially the same pattern of distribution of radioactivity has been observed when the species of experimental animals or the form of $\mathrm{Cr}^{51}$ have been varied. In these confirmatory studies, mice received chromate labelled endotoxin and rabbits chromic $\left(\mathrm{Cr}^{51} \mathrm{Cl}_{3}\right)$ labelled endotoxin $(3,4)$. Attempts are also being made to confirm the in vivo stability of the chromium label by the immunochemical methods customarily employed with simple protein antigens (5). These techniques are of questionable value, however, in the case of endotoxin because its antigenic components do not necessarily correspond to its toxic components. Final proof that $\mathrm{Cr}^{51}$ remains attached in vivo to the toxic portion of the endotoxin complex will depend on recovery from the tissues of $\mathrm{Cr}^{51}$-labelled endotoxin in such a state that the amount of radioactivity per unit of toxicity is equal to or greater than that of the inoculated endotoxin. Until this is done, the possibility must be conceded that endotoxin could be split in vivo and that $\mathrm{Cr}^{51}$ could remain attached to a nontoxic portion.

It is significant that the apparent distribution of chromium-labelled endotoxin obtained from $E$. coli resembles closely that reported after inoculation of iodinated endotoxins from Serratia marcescens and Shigella flexneri $(6,7)$. Seligman, Shear, Leiter, and Sweet (6) found that by far the greatest radioactivity appeared in the liver soon after the intravenous injection of mice with $I^{181}$-labelled endotoxin of $S$. marcescens; and that next to the liver, most radioactivity appeared in the lung and kidney. They did not examine the buffy coat, but reported that radioactivity disappeared so rapidly from the plasma of a rabbit that only 24 per cent of that at zero time was present at four hours. This rapid disappearance from plasma of radioactivity after injection of endotoxin labelled with either $\mathrm{Cr}^{51}$ or $\mathrm{I}^{131}$ is in marked contrast to its slow disappearance after inoculation of iodinated or chromated serum proteins from a heterologous species (8).
The apparent heavy distribution of labelled endotoxin in the liver, buffy coat, lung, and spleen may be related to the ability of their phagocytic cells to remove endotoxin from the circulation. If the endotoxin in those tissues should exert no action locally or systemically, its removal would represent a protective function comparable to that exercised by the same organs when pathogenic bacteria are injected into the circulation. This protective removal of endotoxin might spare such targets as the brain. According to the important evidence of Penner and Klein (9), the brain mediates various systemic effects of endotoxin including hyperglycemia, hemoconcentration, intestinal edema, and even leukopenia. Our failure to detect radioactivity in the brains of rabbits receiving lethal doses of labelled endotoxin would suggest that the amount of endotoxin acting in the brain is too minute to detect, or that an agent which stimulates the brain might be elaborated after localization of endotoxin elsewhere.

It is possible that cerain manifestations may result from injury to those cells in which most of the endotoxin seemed to localize. For example, the apparent localization of chromium labelled endotoxin in the buffy coat, which contains leukocytes and platelets, may be related to the well known changes in leukocyte counts which take place whenever endotoxin is injected. It has also been demonstrated by Berthrong and Cluff (10) that centrifuged neutrophils lose their ability to migrate, and by Stetson (11) that leukocytes and platelets form thrombi in small vessels when rabbits are given endotoxin intravenously. Stetson has suggested that the adhesiveness of leukocytes increases after the injection of endotoxin.

These observations have suggested new lines of investigation which are now in progress : 1) To determine whether the cells which appear to remove endotoxin release substances upon injury which lead to the manifestations characteristic of lethal doses of endotoxin. Among the substances believed to be present in leukocytes are a pyrogen (12), and histamine (13); in hepatic cells, there is said to be both histamine and ferritin, a substance believed capable of inducing irreversible shock $(14,15)$; 2) To determine whether the development of tolerance depends on a greater ability of leukocytes and tissue macrophages to remove endotoxin from the circulation and thus 
protect other targets; and on a greater capacity for the leukocytes themselves to resist its harmful effects. Evidence has already been provided by Cluff (16) that leukocytes can develop such resistance. He observed that centrifuged neutrophils of tolerant animals do not lose their ability to migrate after injection of endotoxin.

\section{SUMMARY}

On the basis of distribution of radioactivity, it appears that labelled endotoxin, after intravenous injection of lethal doses, was present in the plasma, but not erythrocytes, and then rapidly passed mainly to buffy coat and to liver. It accumulated in the buffy coat in high concentration during the period when leukopenia develops. In addition to leukopenia, the period of rapid removal from plasma was accompanied by fever, and sometimes by hypotension and diarrhea. The blood volume, as determined with $\mathrm{Cr}^{51}$-labelled red cells, exhibited a variable response.

\section{REFERENCES}

1. Braude, A. I., Carey, F. J., Sutherland, D., and Zalesky, M., Studies with radioactive endotoxin. I. The use of $\mathrm{Cr}^{\mathrm{at}}$ to label endotoxin of Escherichio coli. J. Clin. Invest., 1955, 34, 850.

2. Grant, R. T., and Rothschild, P., A device for estimating blood-pressure in the rabbit. J. Physiol., 1934, 81, 265.

3. Carey, F. J., Zalesky, M., and Braude, A. I., Studies with radioactive endotoxin. III. Distribution of radioactivity and associated physiologic changes in rabbits injected with lethal and sublethal doses of $E$. coli endotoxin labelled with radioactive chromic chloride. In preparation.

4. Carey, F. J., Zalesky, M., and Braude, A. I., Studies with radioactive endotoxin. IV. Effect of tolerance to $E$. coli endotoxin on the physiologic effects and distribution of radioactivity after injection of $\mathrm{Cr}^{\mathrm{m}}$-labelled endotoxin. In preparation.

5. Gitlin, D., Use of ultraviolet absorption spectroscopy in the quantitative precipitin reaction. J. Immunol., 1949, 62, 437.

6. Seligman, A. M., Shear, M. J., Leiter, J., and Sweet, B., Chemical alteration of polysaccharide from Serratia marcescens. I. Tumor-necrotizing polysaccharide tagged with radioactive iodine. J. Nat. Cancer Inst., 1948, 9, 13.

7. Barnes, F. W., Lupfer, H., and Henry, S. S., The biochemical target of Flexner dysentery somatic antigen. Yale J. Biol. \& Med., 1952, 24, 384.

8. Sterling, $K$. The turnover rate of serum albumin in man as measured by $I^{\text {snd }}$-tagged albumin. $\mathrm{J}$. Clin. Invest., 1951, 30, 1228.

9. Penner, A., and Klein, S. H., The pathogenesis of experimental dysentery intoxication. Production of the lesions by cerebral circulation of the toxin. J. Exper. Med., 1952, 96, 59.

10. Berthrong, M., and Cluff, L., Studies of the effect of bacterial endotoxins on rabbit leukocytes. I. Effect of intravenous injection of the substances with and without induction of the local Shwartzman reaction. J. Exper. Med., 1953, 98, 331.

11. Stetson, C. A., Studies on the mechanism of the Shwartzman phenomenon. J. Exper. Med., 1951, 93, 489.

12. Bennett, I. L., and Beeson, P. B., Studies on the pathogenesis of fever : II. Characterization of feverproducing substances from polymorphonuclear leukocytes and from the fluid of sterile exudates. J. Exper. Med., 1953, 98, 493.

13. Chase, M. W., The allergic state in Bacterial and Mycotic Infections of Man, edited by Dubos, R. J., Philadelphia, J. B. Lippincott, 1948, p. 110-153.

14. Copenhaver, J. H., Jr., Nagler, M. E., and Goth, A., The intracellular distribution of histamine. J. Pharmacol. \& Exper. Therap., 1953, 109, 401.

15. Zweifach, B. W., Functional deterioration of terminal vascular bed in irreversible hemorrhagic shock. Ann. New York Acad. Sc., 1952, 55, 370.

16. Cluff, L. E., Studies of the effect of bacterial endotoxins on rabbit leukocytes. II. Development of acquired resistance. J. Exper. Med., 1953, 98, 349. 